\title{
Synthesis and characterization of $\mathrm{pH}$ and temperature responsive poly(2-hydroxyethyl methacrylate-co-acrylamide) hydrogels
}

\author{
Manuel Rapado ${ }^{1}$ and Carlos Peniche ${ }^{2 *}$ \\ ${ }^{1}$ Center of Technological Applications and Nuclear Development - CEADEN, Havana, Cuba \\ ${ }^{2}$ Center of Biomaterials, University of Havana, Havana, Cuba \\ *peniche@biomat.uh.cu
}

\begin{abstract}
Acrylamide/2-hydroxyethyl methacrylate hydrogels were prepared by simultaneous radiation-induced cross-linking copolymerization of acrylamide (AAm), 2-hydroxyethyl methacrylate (HEMA) and water mixtures at a radiation dose of $10 \mathrm{kGy}$. Hydrogels were characterized by infrared spectroscopy. Dynamic and equilibrium swelling of hydrogels in water and in buffer solutions were investigated. They were sensitive to $\mathrm{pH}$ and temperature. Swelling was non-Fickean and increased with increasing the acrylamide content. Temperature dependence of the equilibrium water uptake of copolymers exhibited a discontinuity around $35{ }^{\circ} \mathrm{C}$ resulting from the weakening of the hydrogen bonds between the hydroxyl groups of HEMA and the amide groups of AAm. The thermodynamic and network parameters derived from swelling and mechanical measurements are compared and discussed. They exhibit a strong dependence on the AAm content in the hydrogel. These hydrogels can be considered for applications in fields requiring environmentally responsive hydrogels such as medicine, pharmacy and bioengeneering.
\end{abstract}

Keywords: acrylamide, hydrogel, hydroxyethyl methacrylate, radiation copolymerization, swelling kinetics.

\section{Introduction}

Hydrogels are three dimensional hydrophilic polymer networks that are capable to absorb considerable amounts of water, but do not dissolve when brought into contact with aqueous solutions ${ }^{[1]}$. In water, hydrogels swell to equilibrium while preserving their shape. The ability to swell and the extent of swelling of hydrogels are governed by two factors: the hydrophilicity of the polymer and the cross-link density of polymer chains ${ }^{[2]}$. Some hydrogels swell and contract in response to external stimulus such as $\mathrm{pH}$, temperature and ionic concentration. These "smart" hydrogels have found a great variety of applications in biomedicine, pharmacy, biotechnology, bioengineering and agriculture, among others.

Hydrogels based on 2-hydroxyethyl methacrylate (HEMA) are usually prepared by bulk polymerisation or polymerisation in solution in the presence of free radical initiators and cross-linking agents ${ }^{[3,4]}$. They have been used in controlled release drug delivery systems, contact lenses, artificial implants, burn dressings, among others due to their biocompatibility, hydrophilicity, softness, and permeability ${ }^{[3]}$. However, in some cases applications are limited due to poor mechanical properties or by low release capacity. Many approaches have been developed to improve their mechanical strength. These include using special co-monomers, changing the type and concentration of the cross-linking agent, optimizing polymerization conditions, forming interpenetrating polymer networks, and radiation cross-linking, among others ${ }^{[5-7]}$. The main advantage of radiation over conventional chemical methods for the preparation of biomaterials relies in its ability to modify macromolecules under fairly mild conditions, without additives. Besides, radiation solves the need of sterilization providing a sterile product without toxic initiator residues ${ }^{[8,9]}$.

Maximum swelling in water of pHEMA is thermodynamically limited to about $40 \%{ }^{[10]}$. Therefore, in order to increase its swelling degree HEMA has been copolymerized with very hydrophilic monomers, such as methacrylic acid (MAAc) ${ }^{[11]}$ and acrylamide (AAm). Copolymers of HEMA and acrylamide (AAm) have been prepared and studied by various authors ${ }^{[1-14]}$. They have shown that there is a significant dependence of swelling on the copolymer composition and that the copolymers prepared are $\mathrm{pH}$ responsive. The mechanical properties of poly(HEMA-co-AAm) hydrogels have been reported before ${ }^{[11,15]}$. However, the results are given for only one HEMA/AAm composition and the copolymers were prepared in presence of a chemical cross-linker.

In order to evaluate the effect of composition on the properties of this copolymer system, in this work we prepare poly(HEMA-co-AAm) hydrogels with different compositions by $\gamma$-irradiation and characterize them in terms of morphology, swelling behaviour and mechanical properties. The thermodynamic and network parameters derived from swelling and mechanical measurements are compared and discussed.

\section{Materials and Methods}

\subsection{Materials}

Acrylamide (AAm), 98.5\% pure, was obtained from BDH (England) and used as received. 2-hydroxyethyl methacrylate (HEMA), 99\% pure was purchased from Fluka (Switzerland). 
HEMA was purified by passing through a column filled with a HQ/MEHQ resin from Polysciences (Eppelheim, Germany) for inhibitor removing, and immediately before use it was purified by vacuum distillation (at $10 \mathrm{~Pa}$ ) at $50^{\circ} \mathrm{C}$. Only the middle fraction collected was used.

\subsection{Copolymer synthesis}

The poly(HEMA-co-AAm) hydrogels were prepared by simultaneous radiation-induced copolymerization and self-bridging of HEMA and AAm in aqueous solution without the use of any cross-linker. The reactants were dissolved in double distilled water. The monomer feed compositions for the hydrogels syntheses are listed in Table 1. The monomer solutions were sealed in $10 \mathrm{~mm}$ diameter and $100 \mathrm{~mm}$ long glass bulbs under $\mathrm{N}_{2} \mathrm{O}$ - saturated atmosphere. They were irradiated with a $10 \mathrm{kGy}$ dose at $25^{\circ} \mathrm{C}$. The dosimetry control for each exposition was determined using Fricke and ceric-cerous dosimetry. Unreacted monomer and uncross-linked polymers were removed by washing the gels for two weeks in distilled water. They were dried to constant weight in vacuum oven at $40{ }^{\circ} \mathrm{C}$.

\subsection{Hydrogel composition}

The chemical composition of hydrogels was calculated from $\mathrm{C}, \mathrm{H}$, and $\mathrm{N}$ elemental microanalysis performed on a CHNS-OEA1108-Elemental Analyzer, from Carlo Erba Instruments (Norwich, UK).

\subsection{Density measurements}

The densities of non-lyophilized hydrogels were determined with a pycnometer using cyclohexane as non-solvent. All density measurements were performed at $25^{\circ} \mathrm{C}$. They were calculated using the following equation ${ }^{[16]}$ :

$$
\rho_{x g}=\frac{m_{x g} \rho_{c y c}}{m_{1}+m_{x g}-m_{2}}
$$

where $m_{\mathrm{xg}}$ is the mass of hydrogel, $m_{1}$ the mass of pycnometer with cycloexane, $m_{2}$ the mass of pycnometer with cycloexane and hydrogel and $\rho_{\text {cyc }}$ the density of cycloexane $\left(0.78 \mathrm{~g} \cdot \mathrm{cm}^{-3}\right)$. Experiments were carried out in triplicate and the mean values for the densities are listed in Table 1.

\subsection{FTIR analysis}

FTIR spectra were obtained with $\mathrm{KBr}$ discs and recording the spectral range from 4000 to $500 \mathrm{~cm}^{-1}$ by using a Nicolet AVATAR 330 Fourier-transformed Infrared
Spectrophotometer (Nicolet Instrument Corp., Madison, WI). Spectra were obtained with a resolution of $2 \mathrm{~cm}^{-1}$ and were averaged over 100 scans. Samples $(5 \mathrm{mg})$ were thoroughly dried and ground with $\mathrm{KBr}$. Discs were prepared by compression under vacuum.

\subsection{Morphologycal analysis}

The morphologies of non-lyophilized and lyophilized hydrogels were determined using an environmental scanning electron microscope (ESEM) FEI Model Quanta 200 Philips/FEI (Redwood City, CA).

\subsection{Swelling experiments}

Dynamic swelling experiments were carried out by placing previously dried hydrogel discs of about $0.7 \mathrm{~cm}$ diameter and $0.2 \mathrm{~cm}$ thick in vials with $50 \mathrm{~mL}$ of double distilled water at $25{ }^{\circ} \mathrm{C}$ or in buffer solutions of various $\mathrm{pHs}(\mathrm{pH} 2,4,6,7.4,8.5,10$, and 12$)$ at $37^{\circ} \mathrm{C}$. The water uptake, $\mathrm{W}$, was calculated by measuring the weight gain of the sample at different times after carefully wiping the surface with a filter paper. It was reported as

$$
W=\frac{m_{t}-m_{0}}{m_{0}}=\frac{m_{t}}{m_{0}}-1
$$

where $m_{\mathrm{t}}$ is the weight of the swollen disc at time $t$ and $m_{0}$ is the weight of the dry disc. All swelling experiments were performed in triplicate.

The water penetration velocity $(v)$ into hydrogels was determined by the weight gain method described by Davidson and Peppas ${ }^{[17]}$. To calculate $v$, Equation 3 was used:

$$
v=\left(\frac{1}{2 A}\right) \times\left(\frac{d W_{g}}{d t}\right)
$$

where $\left(\mathrm{dW}_{\mathrm{g}} / \mathrm{dt}\right)$ is the initial slope of the water uptake versus time curve, $\rho$ denotes is the density of water at the measured temperature, $\mathrm{A}$ is the area of one face of the disc, and the factor 2 accounts for the fact that diffusion takes place through both faces.

\subsection{Preparation of buffer solutions}

Buffers at $\mathrm{pH} 2,4,6,7.4,8.5,10$ and 12 were prepared following the procedure described elsewhere ${ }^{[18]}$. Briefly, $2.4732 \mathrm{~g}$ of boric acid were added to a mixture of $2.3 \mathrm{~mL}$ of glacial acetic acid and $2.7 \mathrm{~mL}$ of phosphoric acid and the

\begin{tabular}{|c|c|c|c|c|c|c|c|}
\hline \multirow[t]{2}{*}{ Sample } & \multicolumn{3}{|c|}{$\begin{array}{l}\text { Reaction mixture composition } \\
(\text { wt- } \%) \\
\end{array}$} & \multicolumn{2}{|c|}{$\begin{array}{c}\text { HEMA } \\
\text { molar fraction }\end{array}$} & \multirow[t]{2}{*}{$\begin{array}{c}\text { Gel fraction } \\
\qquad(\%)\end{array}$} & \multirow[t]{2}{*}{$\begin{array}{c}\text { Polymer } \\
\text { density }\left(\mathrm{g} / \mathrm{cm}^{3}\right)\end{array}$} \\
\hline & HEMA & AAm & $\mathrm{H}_{2} \mathrm{O}$ & Feed & Copolymer* & & \\
\hline polyHEMA & 40 & 0 & 60 & -- & -- & $97 \pm 0.4$ & $1.31 \pm 0.02$ \\
\hline M1 & 40 & 5 & 55 & 0.83 & 0.81 & $99 \pm 0.2$ & $1.36 \pm 0.02$ \\
\hline M2 & 40 & 12 & 48 & 0.63 & 0.65 & $97 \pm 0.3$ & $1.38 \pm 0.04$ \\
\hline M3 & 23 & 18 & 59 & 0.41 & 0.41 & $99 \pm 0.6$ & $1.34 \pm 0.05$ \\
\hline M4 & 10 & 10 & 80 & 0.36 & 0.37 & $97 \pm 0.8$ & $1.27 \pm 0.01$ \\
\hline M5 & 5 & 24 & 71 & 0.10 & 0.11 & $98 \pm 0.5$ & $1.32 \pm 0.02$ \\
\hline polyAAm & 0 & 24 & 76 & -- & -- & $95 \pm 0.3$ & $1.30 \pm 0.05$ \\
\hline
\end{tabular}
resulting solution was diluted with double-distilled water to

Table 1.Composition of the reaction mixtures and gel fraction (\%) for the radiation copolymerization of HEMA and AAm.

*Composition determined by elemental microanalysis using Equation 8 . 
a total volume of $1.0 \mathrm{~L}$. Afterwards $50 \mathrm{~mL}$ portions of this solution were taken, and the $\mathrm{pH}$ was adjusted to the desired $\mathrm{pH}$ by adding an appropriate amount of $0.2 \mathrm{M} \mathrm{NaOH}$.

\subsection{Mechanical properties}

Measurements were performed by imposing a strain on the sample by uniaxial compression and measuring the resulting stress using a texture analyser TA-HD (Stable Micro Systems, UK). The apparatus was equipped with an $18 \mathrm{~mm}$ diameter cylindrical probe running at $0.1 \mathrm{~mm} \cdot \mathrm{s}^{-1}$. All mechanical measurements were conducted in a constant temperature room at $25 \pm 0.1{ }^{\circ} \mathrm{C}$. Hydrogel samples of $18 \mathrm{~mm}$ diameter and 5-6 $\mathrm{mm}$ thick were exposed to unidirectional strain. To this end the gel discs were placed on the plate of the compression cell after gently wiping their surface with filter paper. Strain was applied up to $40 \%$ compression. Measurements were repeated five times on different discs prepared under identical conditions. The stress $(\sigma)$ and strain (ع) were obtained according to Equations 4 and 5.

$$
\begin{aligned}
\sigma & =\frac{P}{A} \\
\varepsilon & =\frac{l_{F}}{l_{0}}
\end{aligned}
$$

Where $P$ represents the compression force, $A$ the area of the cylinder and $l_{0}$ and $l_{F}$ are the initial and final length, respectively. Elasticity modulus (E) values of hydrogels were calculated directly from the initial slope of the stress-strain curves. The average molar mass between cross-links was estimated from the elasticity modulus by the following the equation ${ }^{[16]}$

$$
M_{c}=\frac{3 \rho_{p} R T}{E} \varphi_{2 m}^{1 / 3}
$$

where $\mathrm{R}$ is the gas constant, $\mathrm{T}$ the absolute temperature, $\rho_{\mathrm{p}}$ the polymer density, and $\varphi_{2 M}$ the equilibrium volume fraction of polymer in the swollen state. The volume fraction of the swollen polymer was calculated using the following relation:

$$
\varphi_{m}=\left[1+\frac{\rho_{\mathrm{p}}}{\rho_{\mathrm{s}}}\left(\frac{\mathrm{m}_{\mathrm{s}}}{\mathrm{m}_{0}}\right)-\frac{\rho_{\mathrm{p}}}{\rho_{\mathrm{s}}}\right]^{-1}
$$

In which $\rho_{\mathrm{p}}$ and $\rho_{\mathrm{s}}$ are are the densities of polymer and solvent, respectively, $m_{0}$ and $m_{\mathrm{s}}$ are the mass of polymer before and after swelling, respectively. Determinations were performed at $25^{\circ} \mathrm{C}$.

\section{Results and Discussions}

\subsection{Copolymer composition}

When HEMA/AAm $/ \mathrm{H}_{2} \mathrm{O}$ mixtures are $\gamma$-irradiated free radicals are generated in the aqueous media. Random reactions of these radicals with the monomers lead to the simultaneous polymerization and cross-linking of monomers giving rise to cross-linked poly(HEMA-co-AAm) hydrogels. In this study a series of copolymerization reactions were carried out in water at different monomer feed compositions, while keeping the overall comonomer concentration between 2 and $5 \mathrm{M}$.

For the preparation of mechanically stable hydrogels, the ternary mixtures of HEMA/AAm $/ \mathrm{H}_{2} \mathrm{O}$ were irradiated at a radiation absorbed dose of $10 \mathrm{kGy}$ with a Co-60 gamma source isotope at $25 \pm 1{ }^{\circ} \mathrm{C}$. The copolymerization reaction can be represented, in a simplified form, according to the following equation (Figure 1):

A series of poly(HEMA-co-AAm) hydrogels were obtained by varying the monomer feed weight ratio (HEMA/AAm) from 40:5 to 5:24. The considerations for selecting the feed compositions used in the present work were the solubility of AAm in HEMA and the shape stability of obtained hydrogels in their fully swollen state. The composition of the network chains in the hydrogels was estimated from elemental analysis. The copolymer composition can be represented as $\left(\mathrm{C}_{3} \mathrm{H}_{5} \mathrm{NO}\right)_{X}\left(\mathrm{C}_{6} \mathrm{H}_{10} \mathrm{O}_{3}\right)_{1-X}$, and it can be readily shown that the molar fraction $x$ of AAm in the copolymer can be calculated using the following relation:

$$
\frac{C(\%)}{N(\%)}=\frac{(36-18 x)}{(7 x)}
$$

Where C (\%) and $\mathrm{N}(\%)$ are the corresponding carbon and nitrogen contents in the copolymer as determined by elemental microanalysis. The results are shown in Table 1.

The copolymerization parameters of HEMA (1) with AAm (2) are $r_{1}=1.84$ and $r_{2}=0.41^{[19]}$, but as it can be seen in Table 1, due to the high degrees of conversion attained in the present work, in all cases the copolymer composition is almost the same as that of the feed. As it can be seen in the table, there are no important differences in the samples densities with composition.

\subsection{Spectral characterization}

The infrared spectra of polyHEMA, polyAAm and poly(HEMA-co-AAm) hydrogels M1 and M3 obtained from the corresponding monomer solutions (Table 1) at<smiles>C=CC(N)=O</smiles>

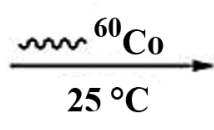

acrylamide (AAm)

\section{2-hydroxyethyl methacrylate (HEMA)}

Figure 1. Schematic representation of the radiation copolymerization reaction of HEMA and AAm.

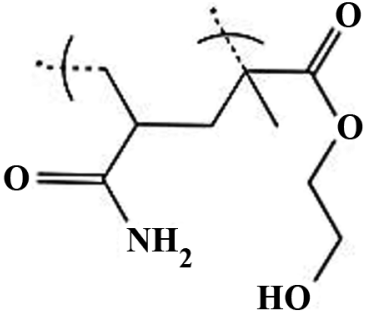

poly(HEMA-co-AAm) 
constant dose of $10 \mathrm{kGy}$ of gamma radiation are shown in Figure 2. Copolymers with other HEMA/AAm compositions exhibited similar qualitative spectra and are not shown. PolyAAm presents a distinctive absorption band at $1657 \mathrm{~cm}^{-1}$ $(\mathrm{C}=\mathrm{O}$ stretching, amide $\mathrm{I})$. The $\mathrm{N}-\mathrm{H}$ stretching absorbs in the range $3500-3100 \mathrm{~cm}^{-1}$ and the band at $1460 \mathrm{~cm}^{-1}$ was associated with the stretching vibration of acyl amino groups $^{[20]}$. On the other hand, polyHEMA exhibits a sharp absorption band at $1730 \mathrm{~cm}^{-1}$, which is characteristic of the $\mathrm{C}=\mathrm{O}$ stretching. The absorption band at $2950 \mathrm{~cm}^{-1}$ with a weak shoulder is attributed to $\mathrm{C}-\mathrm{H}$ stretching vibration of methylene groups. The wide $\mathrm{O}-\mathrm{H}$ stretching absorption band appearing at $3500-3220 \mathrm{~cm}^{-1}$ indicates the presence of hydrogen bonding between hydroxyl groups of polyHEMA ${ }^{[14]}$.

The IR spectrum of M1 and M3 copolymers exhibit all the characteristic bands of the corresponding homopolymers. It is worth mentioning the appearance of a broad absorption band peaking at $3220 \mathrm{~cm}^{-1}$ in the spectrum of the copolymers indicating the formation of hydrogen bonding, most probably between the $-\mathrm{OH}$ groups of HEMA and the $-\mathrm{NH}_{2}$ of the amide groups of AAm. The absorption bands observed at 1277 and $1460 \mathrm{~cm}^{-1}$, characteristic of the methyl and amide groups of HEMA and AAm, respectively, are also present in the spectra of $\mathrm{M} 1$ and $\mathrm{M} 3^{[14]}$. It can be appreciated that the relative intensity of the main polyAAm absorption bands are greater in the acrylamide richer copolymer M3.

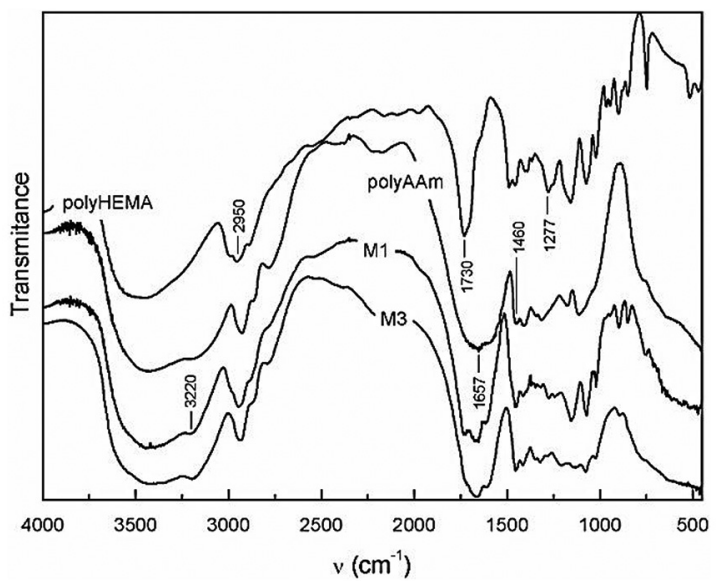

Figure 2. FTIR spectra of polyHEMA, polyAAm and poly(HEMA-co-AAm) hydrogels M1 and M3.

\subsection{Swelling of hydroge/s}

A very important intrinsic characteristic property of hydrogels is their ability to swell when immersed in water or aqueous solutions ${ }^{[2]}$.In the present study the effect of copolymer composition, $\mathrm{pH}$ and temperature on swelling for HEMA/AAm copolymers obtained by irradiation at 10 kGy were investigated.

\subsubsection{Swelling as function of copolymer composition}

Swelling of polyHEMA, polyAAm and poly(HEMA-co-AAm) hydrogels of various compositions (Table 1) was followed at $25{ }^{\circ} \mathrm{C}$ in water for a long time to ensure equilibrium. The resulting swelling curves are shown in Figure 3 and the equilibrium water uptakes for all compositions are listed in Table 2, where a strong dependence of the swelling degree on composition is observed.

As expected, polyAAm experienced higher maximum swelling $(21.25 \mathrm{~g} / \mathrm{g})$ than polyHEMA $(1.023 \mathrm{~g} / \mathrm{g})$, due to the greater hydrophilicity of the former. It has been pointed out that the low swelling of polyHEMA is due to intra- and intermolecular hydrogen bonding. In HEMA-rich copolymers swelling is controlled by the intermolecular hydrogen bonding between hydroxyl and amide groups and intramolecular hydrogen bonding between amide groups $^{[12]}$. As the copolymers became richer in AAm, equilibrium swelling increased from $1.444 \mathrm{~g} / \mathrm{g}$ for M1

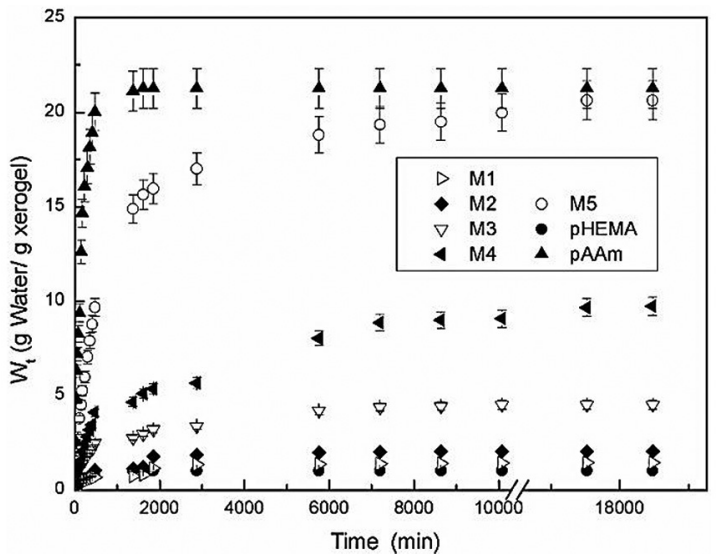

Figure 3. Swelling at $25{ }^{\circ} \mathrm{C}$ of polyHEMA, polyAAm and poly(HEMA-co-AAm) hydrogels discs of different compositions (Table 1).

Table 2. Equilibrium water uptake and diffusion parameters of polyHEMA, polyAAm and poly(HEMA-co-AAm) hydrogels discs of different compositions (see Table 1).

\begin{tabular}{|c|c|c|c|c|c|}
\hline \multirow[t]{2}{*}{ Polymer } & \multirow{2}{*}{$\begin{array}{c}\mathrm{W}_{\infty} \\
\left(\mathrm{gH}_{2} \mathrm{O} / \mathrm{g} \text { polymer }\right)\end{array}$} & \multirow{2}{*}{$\begin{array}{l}\text { Penetration velocity } \\
\qquad\left(\times 10^{-5} \mathrm{~cm} / \mathrm{s}\right)\end{array}$} & \multicolumn{2}{|c|}{$\begin{array}{c}\text { Diffusion parameters } \\
\text { (Equation 14) }\end{array}$} & \multirow{2}{*}{$\begin{array}{c}\mathrm{D} a \\
\left(\mathrm{~cm}^{2} / \mathrm{s}\right)\end{array}$} \\
\hline & & & $n$ & $\left(k \times 10^{2}\right)$ & \\
\hline polyHEMA & 1.023 & $3.65 \pm 0.28$ & 0.55 & 2.9 & $2.188 \times 10^{-6}$ \\
\hline M1 & 1.444 & $1.07 \pm 0.25$ & 0.48 & 2.4 & $1.241 \times 10^{-7}$ \\
\hline M2 & 2.364 & $2.93 \pm 0.55$ & 0.74 & 0.44 & $1.630 \times 10^{-7}$ \\
\hline M3 & 4.504 & $3.75 \pm 0.27$ & 0.56 & 1.7 & $3.451 \times 10^{-7}$ \\
\hline M4 & 9.776 & $3.90 \pm 0.16$ & 0.59 & 1.2 & $4.159 \times 10^{-7}$ \\
\hline M5 & 20.62 & $5.55 \pm 0.38$ & 0.66 & 0.77 & $4.651 \times 10^{-7}$ \\
\hline polyAAm & 21.25 & $9.57 \pm 0.22$ & 0.66 & 0.20 & $5.312 \times 10^{-6}$ \\
\hline
\end{tabular}


(HEMA/AAm 40:5) to 20.62 for M5 (HEMA/AAm 5:24). It is interesting to note in Table 2 that the initial solvent penetration velocity is higher in polyHEMA than in the copolymers with low AAm content (M1 and M2). This indicates that hydrogels of these two copolymers have a more compact structure than polyHEMA, which hinders the penetration of water during the initial swelling stage.

Swelling kinetics of HEMA/AAm hydrogels obtained by $\gamma$-irradiation was evaluated using the Ritger-Peppas equation ${ }^{[22]}$ (Equation 9) in order to describe the mechanism of water diffusion in the gels.

$$
\frac{W_{t}}{W_{\infty}}=k t^{n}
$$

where $\mathrm{M}_{t} / \mathrm{M}_{\infty}$ is the fractional water uptake, $k$ is a kinetic constant, $t$ is the swelling time and $n$ is the diffusional exponent that can be related to the solvent transport mechanism. For a cylindrical sample, when $n=0.45$, the water transport mechanism is Fickian diffusion. When $n=1$, Case II transport occurs, leading to zero-order release. When the value of $n$ is between 0.45 and 1 , anomalous (non-Fickian) transport is observed. This mathematical model is valid only for the first $60 \%$ of the total solvent uptake. The values of the diffusional exponents, $n$ and diffusion constants, $k$, can be calculated from slope and intercept of the logarithmic plot of the data in Figure 4 according to:

$$
\ln \frac{W_{t}}{W_{\infty}}=\ln k+n \ln t
$$

They are reported in Table 2 . In all cases the diffusional exponent $n>0.45$, is indicating that the transport mechanism is non-Fickean. This anomalous behaviour was also obtained for HEMA/AAm hydrogels prepared by redox polymerization employing N,N'-mehthylenebisacrylamide as cross-linker. It is generally attributed to a slow relaxation rate of the polymer matrix ${ }^{[12]}$.

The estimation of diffusion coefficients for non Fickiansorption processes is not straightforward. However, reasonably values of diffusion coefficients can be obtained by using the following simple equation ${ }^{[23]}$ :

$$
D a=\frac{0.049}{\left(t / 4 l^{2}\right)_{1 / 2}}
$$

where $D a$ is the apparent average diffusion coefficient in $\mathrm{cm}^{2} \cdot \mathrm{s}^{-1}, l$ is one half of the cylindrical sample diameter in $\mathrm{cm}, t$ is the time at which the swelling is one half the equilibrium value $\left(\mathrm{M}_{\mathrm{t}} / \mathrm{M}_{\infty}=0.5\right)$. The $\mathrm{D} a$ values reported in Table 2 are approximately one order of magnitude smaller for the copolymers as compared to the respective homopolymers. Probably the intramolecular bonding between the hydroxyl groups of HEMA and the amide groups of AAm in the copolymers are responsible for this decrease in $\mathrm{D} a$. However, the general trend of the apparent average diffusion coefficients is to increase as the AAm content in the copolymer hydrogels increases.

The $\mathrm{pH}$ dependence of the swelling capacity of polyHEMA, polyAAm, M1, M3, M4 and M5 hydrogels obtained with an irradiation dose of $10 \mathrm{kGy}$ was evaluated in a wide $\mathrm{pH}$ interval at constant ionic strength $(\mathrm{I}=0.72 \mathrm{M})$ and $37^{\circ} \mathrm{C}$. The equilibrium water uptakes for each composition and $\mathrm{pH}$ are shown in Figure 4. The water uptake of polyHEMA was small and almost $\mathrm{pH}$ independent in the whole $\mathrm{pH}$ interval studied, whereas the swelling degree of polyAAm was much higher and increased with increasing $\mathrm{pH}$. This increase became more prominent as the $\mathrm{pH}$ raised from 6 to 12 . This is the result of the basic hydrolysis of the amide groups of acrylamide, with formation of carboxylate ions. These negatively charged carboxylate ions are highly solvated and the repulsion between them provokes the expansion of the hydrogel network with the consequent increase in water uptake. As expected, the swelling capacity of the copolymer hydrogels increased with increasing their AAm content and they all exhibited the same basic hydrolysis effect on swelling at $\mathrm{pH}$ higher than 6 , which became more significant as the AAm content in the hydrogel increased. A similar hydrolysis effect on swelling has been reported for other acrylamide based hydrogels ${ }^{[24]}$.

An interesting feature of the system studied that has not been previously reported is the temperature dependence of $\mathrm{W}_{\infty}$, the equilibrium water uptake of poly(HEMA-co-AAm) hydrogels. A plot of $\ln \mathrm{W}_{\infty}$ against the reciprocal of the swelling temperature is shown in Figure 5. Two straight lines with different slopes are obtained for all hydrogels in the

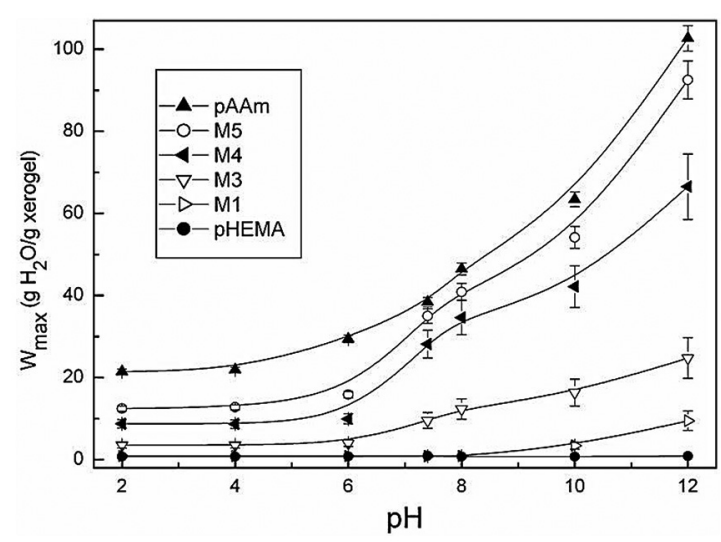

Figure 4. Equilibrium water content of polyHEMA, polyAAm and $\mathrm{M} 1, \mathrm{M} 3, \mathrm{M} 4$ and M5 hydrogels at different $\mathrm{pH}$ values at constant ionic strength $(\mathrm{I}=0.72 \mathrm{M})$ and $37^{\circ} \mathrm{C}$.

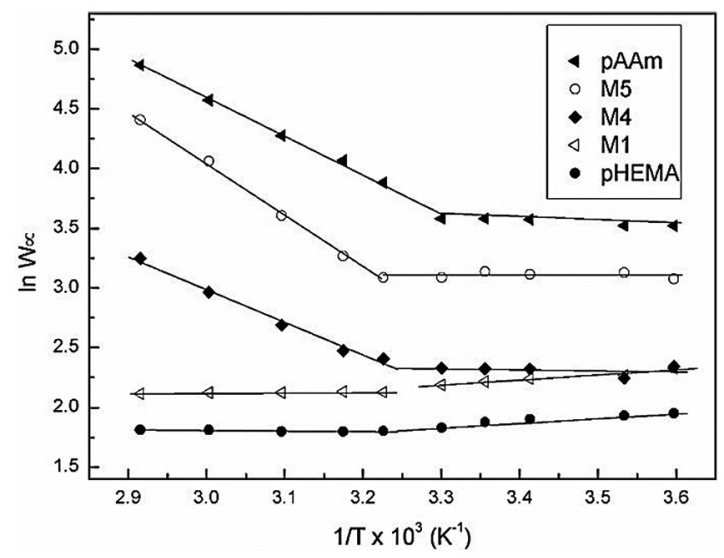

Figure 5. Temperature dependence of the equilibrium water content, $\mathrm{W}_{\infty}$, for poly HEMA, polyAAm and M1, M4 and M5 copolymers. 
temperature range studied. This behavior can be analyzed taking into consideration the Gibbs-Helmholtz equation for the infinite or equilibrium water content ${ }^{[25]}$ :

$$
\frac{d \ln \left(W_{\infty}\right)}{d(1 / T)}=-\frac{\Delta H_{m}}{\mathrm{R}}
$$

where $\mathrm{R}$ is the gas constant and $\Delta \mathrm{H}_{\mathrm{m}}$ is the enthalpy of mixing between the dry polymer and an infinite amount of water. The positive slope of the straight lines observed in Figure 5 for polyHEMA and M1 indicates an exothermic mixing process. Using Equation 12 the values of $\Delta \mathrm{H}_{\mathrm{m}}$ were obtained. They are listed in Table 3 where it can be appreciated that the absolute values of $\Delta \mathrm{H}_{\mathrm{m}}$ in the low temperature range $\left(\mathrm{T}<35^{\circ} \mathrm{C}\right)$ are quite small. The small negative values of the enthalpy of mixing obtained for polyHEMA and M1 have been reported before for this homopolymer ${ }^{[26]}$ and its copolymers with hydrophobic monomers ${ }^{[26,27]}$.

The sudden change in the slope of the straight lines is interpreted in terms of the weakening of the hydrogen bonds between the $\mathrm{OH}$ the hydroxyl groups of HEMA and the amide groups of AAm in the copolymers. These hydrogen bonds act as physical cross-links that strengthen the structure and oppose swelling. Apparently above $35^{\circ} \mathrm{C}$ the thermal energy is sufficiently high to lose these bonds allowing increasing the water uptake capacity of acrylamide and acrylamide richer copolymer hydrogels. A different swelling-temperature behaviour of HEMA/AAm hydrogels prepared by $\gamma$-irradiation at $50 \mathrm{kGy}$ in the presence of trithioglycolic acid (TTGA) was reported by El-Din and El-Naggar ${ }^{[14]}$. In contrast with the present findings their hydrogels shrank by heating above $25-30{ }^{\circ} \mathrm{C}$. They concluded that these systems posses a lower critical solution temperature (LCST) at about $25^{\circ} \mathrm{C}$ as a result of the formation of hydrophobic interchain bonding by copolymerization between TTGA and AM or HEMA.

\subsection{Solubility parameters of HEMA/AAm copolymer hydrogels}

The solubility parameter $(\delta)$ is an important indicator to express the interaction between a polymer and a solvent. The solubility parameter, $\delta_{\mathrm{p}}$, of a given poly(HEMA-co-AAm) hydrogel was taken as that of the solvent mixture that gives the maximum swelling degree for the network. To this end mixtures of various compositions of water $\left(\delta_{1}=23.40\right.$ $\left.\left(\mathrm{cal} \mathrm{cm}^{-3}\right)^{1 / 2}\right)$ and dimethyl formamide $\left(\delta_{2}=12.13\left(\mathrm{cal} \mathrm{cm}^{-3}\right)^{1 / 2}\right)$ were prepared. The solubility parameter of the mixture $\left(\delta_{\mathrm{m}}\right)$ was calculated using the formula:

$$
\delta_{m}=\frac{x_{1} V_{1} \delta_{1}+x_{2} V_{2} \delta_{2}}{x_{1} V_{1}+x_{2} V_{2}}
$$

Where $x_{\mathrm{i}}, V_{I}$ and $\delta_{\mathrm{i}}$ are the molar fraction, molar volume, and solubility parameter of component $i$, respectively $\left(V_{l}=18.02 \mathrm{~cm}^{-3} \mathrm{~mol}^{-1}\right)$.

Table 3. Values of the enthalpy of mixing $\Delta H_{m}$ for polyHEMA, poly AAM and M1, M4 and M5 copolymers.

\begin{tabular}{cccccc}
\hline $\begin{array}{c}\Delta \mathbf{H}_{\mathbf{m}} \\
\left(\mathbf{k J} \cdot \mathbf{m o l}^{-\mathbf{1}}\right)\end{array}$ & polyHEMA & M1 & M4 & M5 & polyAAm \\
\hline Temp $<35^{\circ} \mathrm{C}$ & -3.2 & -3.5 & 0.74 & 0.00 & 2.1 \\
$\mathrm{Temp}>35^{\circ} \mathrm{C}$ & 0.32 & -0.38 & 23.3 & 36.6 & 27.1 \\
\hline
\end{tabular}

The equilibrium swelling of samples M1, M3, M4 and M5 in the various solvent mixtures prepared is represented in Figure 6 as a function of the solubility parameter $\delta_{\mathrm{m}}$. The value of $\delta_{\mathrm{m}}$ corresponding to maximum swelling gives the solubility parameter, $\delta_{\mathrm{p}}$, of the respective copolymer. These are listed in Table 4.

Swelling experiments allow calculating the molecular weight between cross-links, $M_{c}$, which is the basic parameter that characterizes the structure of a hydrogel network. This parameter describes the average molecular weight of polymer chains between two consecutive junctions. These junctions include chemical cross-links, physical entanglements, crystalline regions, and polymer complexes (hydrogen bonding, salt bonds). $M_{c}$ strongly influences the physical and mechanical properties of the hydrogel. Therefore, its determination is of great practical significance. It can be calculated according to the Flory-Rehner equation for the swelling of a perfect network ${ }^{[28,29]}$ :

$$
M_{c}=-\rho_{p} V_{1} \frac{(1-2 / f) \varphi_{2 r}^{2 / 3} \varphi_{2 m}^{1 / 3}}{\ln \left(1-\varphi_{2 m}\right)+\varphi_{2 m}+\chi \varphi_{2 m}^{2}}
$$

In Equation $14 \rho_{P}$ is the density of the polymer $\left(\right.$ in $\mathrm{g} \cdot \mathrm{cm}^{-3}$ ), $V_{1}$ is the molar volume of the swelling solvent $\left(\mathrm{in}^{3} \cdot \mathrm{mol}^{-1}\right.$ ), $\varphi_{2 \mathrm{~m}}$ is the volume fraction of the cross-linked polymer in the swollen gel polymer, $\varphi_{2 \mathrm{r}}$ is the polymer volume fraction in the relaxed state, i.e. after cross-linking but before swelling, $f$ is the cross-linking functionality $\chi$ is the Flory-Huggins interaction parameter, between the polymer and solvent. The value of $M_{c}$ determined from swelling data will be represented as $\left(\mathrm{M}_{\mathrm{c}}\right)_{\mathrm{s}}$.

Another important parameter describing the hydrogel network properties is the effective cross-link density, $v_{\mathrm{e}}$ which can be calculated as:

$$
v_{e}=\rho_{p} / M_{c}
$$

The value of $v_{\mathrm{e}}$ determined from swelling data will be represented as $\left(v_{\mathrm{e}}\right)_{\mathrm{s}}$. The Flory-Huggins interaction parameter $\chi$ between water and the hydrogel can be expressed in

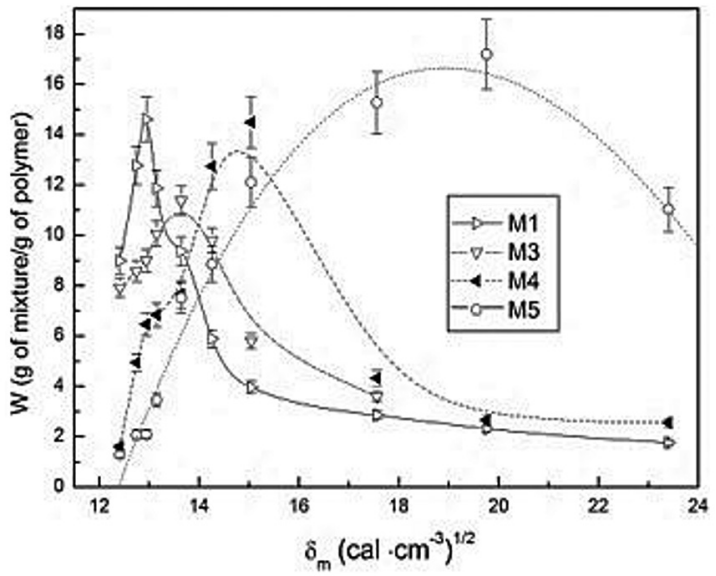

Figure 6. Experimental values of maximum swelling of the HEMA/AAm copolymers hydrogels M1, M3, M4 and M5 as a function of the solubility parameter of the solvent mixture. 
terms of the solubility parameters of the polymer $\left(\delta_{\mathrm{p}}\right)$ and the solvent $\left(\delta_{1}\right)$ :

$$
\chi=\frac{V_{1}\left(\delta_{p}-\delta_{1}\right)^{2}}{R T}+0.34
$$

where $\mathrm{R}$ is the gas constant and $\mathrm{T}$ is the absolute temperature ${ }^{[30]}$. The interaction parameter $\chi$ is also frequently calculated from the simple equation:

$$
\chi_{a p} \cong \frac{1}{2}+\frac{\varphi_{2 m}}{3}
$$

Equation 17 is derived from Equation 14 assuming that $M_{c}$ goes to infinity ${ }^{[15]}$. The values of the solubility parameters and the parameter $\chi$ of the poly(HEMA-co-AAm) hydrogels are listed in Table 4 . The great similarity between the values of $\chi$ obtained by Equations 16 and 17 is apparent.

Table 4 illustrates that there is a clear dependence of both, the solubility parameter and $\chi$ on the copolymer composition. As the copolymer becomes richer in the more hydrophilic component, AAm, $\delta_{\mathrm{p}}$ increases. The increase in $\delta_{\mathrm{p}}$ causes a decrease in the polymer-solvent interaction parameter $\chi$ indicating a greater water compatibility of the copolymer hydrogels richer in AAm. This is in agreement with the composition dependence of the equilibrium water uptake values of poly(HEMA-co-AAm) hydrogels shown in Table 2. The $\chi$ values reported for the poly(HEMA-co-AAm) hydrogels lie in between those reported for polyAAm $(0.44-0.495)^{[3]}$ and polyHEMA $(0.77-0.83)^{[32]}$. Mahmudi et al. ${ }^{[15]}$ studied the effect of cross-linker composition on molecular parameters of a poly(HEMA-co-AAm) hydrogel obtained by $\gamma$-irradiaton with a $6.6 \mathrm{kGy}$ dose using methylenebisacrylamide as cross-linker. They reported $\chi$ values of $0.56-0.57$, almost independent of the amount of cross-linker used. This value is higher than those reported in Table 4. However, the monomer composition of their reaction mixture (HEMA/AAm $/ \mathrm{H}_{2} \mathrm{O} \approx 25: 25: 50$ ) and the radiation dose were different from those of the present work (see Table 1). In addition they do not report the temperature of experiments, which precludes comparison.

\subsection{Mechanical behavior of hydrogels}

The effect of composition on the mechanical properties of poly(HEMA-co-AAm) hydrogels was investigated by uniaxial compresion tests in order to evaluate the true $\mathrm{M}_{\mathrm{c}}$ value, the interaction parameter $\chi$ and the effective cross-link density, $v_{\mathrm{e}}$. The subscript " $m$ " will be used here to indicate that these parameters are calculated from mechanical measurements. Typical stress-strain curves of hydrogels swelled to equilibrium are given in Figure 7. As it can be seen in the figure, there is a marked dependence of the mechanical response on hydrogels composition. The magnitude of stress increased with increasing HEMA content in the copolymer hydrogel for a given strain. Elasticity modulus (E) values of hydrogels were calculated from slope of the initial linear segment of the stress-strain curves. It was then possible to calculate $M_{c}$ (Equation 6) after evaluating $\varphi_{\mathrm{m}}$ using Equation 7.

It has been stated that simple compression analyses can be used for the determination of effective cross-linking density without needing polymer-solvent based parameters as in the case of swelling ${ }^{[33]}$. The effective cross-linking density $\left(v_{\mathrm{e}}\right)$ of the poly(HEMA-co-AAm) hyrogels were obtained from compression-strain measurements combining Equations 6 and 15:

$$
v_{e}=\frac{E \varphi_{2 m}^{-1 / 3}}{3 R T}
$$

The network parameters of hydrogels obtained from mechanical measurements are listed in Table 5. They exhibit the same tendency as the values obtained from swelling measurements, in that $v_{e}$ decreases and $M_{c}$ increases with increasing the acrylamide content of hydrogels. A very good agreement is also found between the values of the Flory-Huggins interaction parameters $\chi$ evaluated from solubility parameters (Equation 16) and those obtained from mechanical measurements. However, the values of molecular weight between cross-links, $M_{c}$, calculated from swelling experiments are about 3 to 4 times higher than those obtained from mechanical measurements. This discrepancy has been obtained before for other acrylamide based copolymers ${ }^{[15]}$, and it has been explained in terms of the high sensitivity of the denominator in Equation 14 to the value of $\chi$ for highly swelled gels $\left(\varphi_{2 M}<<1\right)$. Therefore, even though the $M_{c}$ values obtained from Equation 14 allow knowing the correct tendency of the variation of the molecular weight between cross-links with the copolymer composition, the more reliable $M_{c}$ values should be the ones obtained from mechanical measurements.

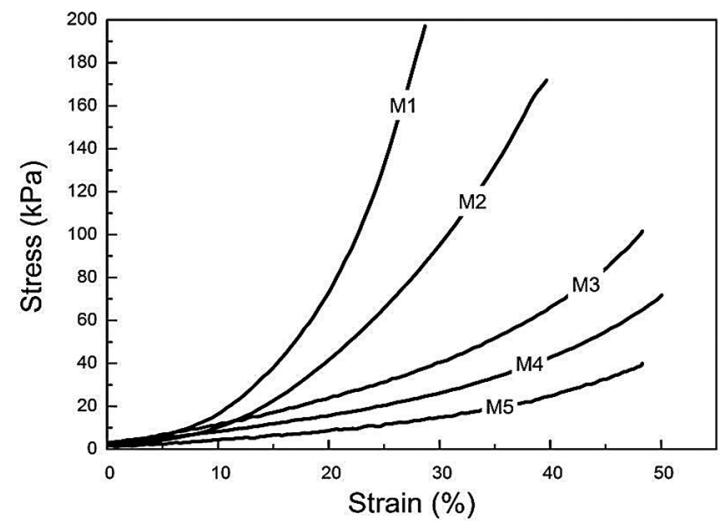

\begin{tabular}{|c|c|c|c|c|c|}
\hline Copolymer & $\delta_{p}\left(\mathrm{cal}^{\prime} \cdot \mathrm{cm}^{-3}\right)^{1 / 2}$ & $(\chi)_{s}$ & $(\chi)_{\mathrm{ap}}$ & $\begin{array}{c}\left(\mathrm{M}_{\mathrm{c}}\right)_{\mathrm{s}} \times 10^{-5} \\
\left(\mathrm{~g} \cdot \mathrm{mol}^{-1}\right)\end{array}$ & $\left(v_{\mathrm{e}}\right)_{\mathrm{s}} \times 10^{5}\left(\mathrm{~mol} \cdot \mathrm{cm}^{-3}\right)$ \\
\hline M1 & 12.93 & 0.54 & 0.537 & 3.154 & 1.27 \\
\hline M3 & 13.63 & 0.52 & 0.522 & 2.655 & 1.51 \\
\hline M4 & 15.06 & 0.51 & 0.511 & 2.539 & 2.17 \\
\hline M5 & 18.96 & 0.50 & 0.508 & 1.817 & 3.51 \\
\hline
\end{tabular}

Figure 7. Strain-stress curves of poly(HEMA-co-AAm) hydrogels.

Table 4. Solubility parameters and Flory-Huggins interaction parameter and network parameters of poly(HEMA-co-AAm) hydrogels. 
Table 5. Network parameters from stress-strain measurements.

\begin{tabular}{|c|c|c|c|c|c|c|}
\hline Sample & $\mathrm{E}(\mathrm{kPa})$ & $\begin{array}{l}\left(v_{\mathrm{e}}\right)_{M} \times 10^{5} \\
\left(\mathrm{~mol} \cdot \mathrm{cm}^{-3}\right)\end{array}$ & $\begin{array}{c}\left(\mathrm{M}_{\mathrm{c}}\right)_{M} \times 10^{-5} \\
\left(\mathrm{~g} \cdot \mathrm{mol}^{-1}\right) \\
\end{array}$ & $\left(\mathbf{M}_{\mathrm{c}}\right)_{\mathrm{s}} /\left(\mathbf{M}_{\mathrm{c}}\right)_{M}$ & $(\chi)_{M}$ & $(\chi)_{S}-(\chi)_{M}$ \\
\hline pHEMA & 1.776 & 1.03 & 1.250 & --- & 0.59 & --- \\
\hline M1 & 0.861 & 1.06 & 1.063 & 2.96 & 0.55 & -0.01 \\
\hline M2 & 0.558 & 1.07 & 1.032 & --- & 0.54 & --- \\
\hline M3 & 0.431 & 1.71 & 0.664 & 4.00 & 0.52 & 0.00 \\
\hline M4 & 0.297 & 2.05 & 0.523 & 4.85 & 0.51 & 0.00 \\
\hline M5 & 0.176 & 2.93 & 0.434 & 4.19 & 0.50 & 0.00 \\
\hline pAAm & 0.174 & 4.49 & 0.253 & ---- & 0.50 & ---- \\
\hline
\end{tabular}

\section{Conclusions}

Temperature and $\mathrm{pH}$ sensitive poly(HEMA-co-AAm) hydrogel networks were synthesized by $\gamma$-irradiation of aqueous solutions of acrylamide and 2-hydroxyethyl methacrylate at a $10 \mathrm{kGy}$ dose.

FTIR spectra indicate the formation of hydrogen bonding between the $-\mathrm{OH}$ groups of HEMA and the $-\mathrm{NH}_{2}$ groups of AAm. Water diffusion to poly(HEMA-co-AAm) hydrogels is non-Fickean. Swelling is sensitive to $\mathrm{pH}$ and temperature and increases with increasing the AAm content in the hydrogel. At $\mathrm{pH}$ values above 6 swelling of copolymers increases considerable with increasing $\mathrm{pH}$ due to partial hydrolysis of the acrylamide moieties. The temperature dependence of swelling exhibits a marked change around $30-35^{\circ} \mathrm{C}$ that becomes more prominent for copolymers richer in AAm, which were attributed to the weakening of the hydrogen bonding between the $-\mathrm{OH}$ groups of HEMA and the $-\mathrm{NH}_{2}$ of AAm. The solubility parameter of copolymers, the cross-link density of copolymers and the elastic modulus are also dependent of the AAm content of the hydrogels.

It can be concluded that swelling and mechanical properties of poly(HEMA-co-AAm) copolymers prepared by $\gamma$-irradiation can be tailored to meet specific requirements for applications in different fields requiring environmentally responsive hydrogels such as medicine, pharmacy and bioengeneering.

\section{Acknowledgments}

The authors thanks Prof. Januz M. Rosiak, for his valuable help, discussions and remarks checking the manuscript. Part of the work was done in the framework of fellowships at the Institute of Applied Radiation Chemistry, Technical University of Lotdz, Poland. We also wish to thank Lic. Rosalba ZayasMarín for her help in checking the English spelling of this article.

\section{References}

1. Ratner, B. R., Hoffman, A. S., Schoen, F. J., \& Lemons, J. E. (Ed.). (2013). Biomaterials science. An introduction to materials in medicine. Oxford: Academic Press.

2. Rosiak, J. M., Ulanski, P., Pajewski, L. A., Yoshii, F., \& Makuuchi, K. (1995). Radiation fromation of hydrogels for biomedical purposes. Some remarks and comments. Radiation Physics and Chemistry, 46(2), 161-168. http://dx.doi.org/10.1016/0969806X(95)00007-K.
3. Witchterle, O., \& Lím, D. (1960). Hydrophilic gels for biological use. Nature, 185(4706), 117-118. http://dx.doi. org/10.1038/185117a0.

4. Barbucci, R. (Ed.). (2009). Hydrogels. Biological properties and applications. MIlan: Springer.

5. Lou, X., \& van Coppenhagen, C. (2001). Mechanical characteristics of poly(2-hydroxyethyl methacrylate) hydrogels crosslinked with various difunctional compounds. Polymer International, 50(3), 319-325. http://dx.doi.org/10.1002/pi.630.

6. Park, S., Nam, S. H., \& Koh, W. G. (2012). Preparation of collagen-immobillized poly(ethylene glycol/poly(2-hydroxyethyl methacryalte) Interpenetrating network hydrogels for potential application of artificial cornea. Journal of Applied Polymer Science, 123(2), 637-645. http://dx.doi.org/10.1002/app.34532.

7. Ramaraj, B., \& Radhakrishnan, G. (1994). Modification of the dynamic swelling behaviour of poly(2-hydroxyethyl methacrylate) hydrogels in water through interpenetrating polymer networks (IPNs). Polymer, 35(10), 2167-2173. http:// dx.doi.org/10.1016/0032-3861(94)90245-3.

8. Chapiro, A. (1995). Radiation chemistry in the field of biomaterials. Radiation Physics and Chemistry, 46(2), 159160. http://dx.doi.org/10.1016/0969-806X(95)00006-J.

9. Rosiak, J. M., \& Yoshii, F. (1999). Hydrogels and their medical applications. Nuclear Instruments and Methods in Physics Research Section B, 151(1-4), 56-64. http://dx.doi.org/10.1016/ S0168-583X(99)00118-4.

10. Macret, M., \& Hild, G. (1982). Hydroxyalkyl methacrylates: hydrogel formation based on the radical copolymerization of 2-hydroxyethylmethacrylate and 2,3-dihydroxypropylmethacrylate. Polymer, 23(5), 748-753. http://dx.doi.org/10.1016/00323861(82)90063-5.

11. Dušek, K., \& Janáček, J. (1975). Hydrophilic gels based on copolymers of 2-hydroxyethyl methacrylate with methacrylamide and acrylamide. Journal of Applied Polymer Science, 19(11), 3061-3075. http://dx.doi.org/10.1002/app.1975.070191111.

12. Işik, B. (2000). Swelling behaviour of acrylamide- 2 hydroxyethyl methacrylate hydrogels. Turkish Journal of Chemistry, 24, 147156. Retrieved in 16 July 2013, from http://journals.tubitak. gov.tr/chem/issues/kim-00-24-2/kim-24-2-5-98080.pdf

13. Singh, B., Chauhan, N., Kumar, S., \& Bala, R. (2008). Psyllium and copolymers of 2-hydroxyethylmethacrylate and acrylamide -based novel devices for the use in colon specific antibiotic drug delivery. International Journal of Pharmaceutics, 352(12), 74-80. http://dx.doi.org/10.1016/j.ijpharm.2007.10.019. PMid:18055144.

14. El-Din H. M. N., \& El-Naggar, A. W. M. (2004). Synthesis and characterization of hydroxyethyl methacrylate/acrylamide responsive hydrogels. Journal of Applied Polymer Science, 95(5), 1105-1115.

15. Mahmudi, N., Şen, M., Rendevski, S., \& Güven, O. (2007). Radiation synthesis of low swelling acrylamide based hydrogels and determination of average molecular weight 
between cross-links. Nuclear Instruments and Methods in Physics Research Section B, 265(1), 375-378. http://dx.doi. org/10.1016/j.nimb.2007.09.007.

16. Iza, M., Stoianovici, G., Viora, L., Grossiord, J. L., \& Couarraze, G. (1998). Hydrogels of poly(ethylene glycol): mechanical characterization and release of a model drug. Journal of Controlled Release, 52(1-2), 41-45. http://dx.doi.org/10.1016/ S0168-3659(97)00191-0. PMid:9685934.

17. Davidson, G. W. R., 3rd, \& Peppas, N. A. (1986). Solute and penetrant diffusion in swellable polymers. V. Relaxation -controlled transport in P (HEMA-cp-MMA) copolymers. Journal of Controlled Release, 3(1-4), 243-258. http://dx.doi. org/10.1016/0168-3659(86)90096-9.

18. Lurie, Y. Y. (1979). Handbook of analytical chemistry. Moscow: Chemistry Press.

19. Ham, G. E. (1966). Copolymerization. In H. F. Mark, N. Gaylord, $\&$ N. M. Bikales (Ed.), Encyclopedia of polymer science and technology (Vol. 4, pp. 165-244). New York: Interscience.

20. Murugan, R., Mohan, S., \& Bigotto, A. (1998). FTIR and polarised raman spectra of acrylamide and polyacrylamide. Journal of the Korean Physical Society, 32(4), 505-512. Retrieved in 13 May 2013, from http://www.kps.or.kr/jkps/ downloadPdf.asp?articleuid=\%7B43E6F355-D43F-4D4EB386-6E5B29A6F648\%7D

21. Bell, C. L., \& Peppas, N. A. (1995). Biomedical membranes from hydrogels and interpolymer complexes. In N. A. Peppas, \& R. S. Langer (Ed.), Biopolymers II (Advances in Polymer Science, Vol. 122, pp. 125-175). Berlin: Springer-Verlag.

22. Ritger, P. L., \& Peppas, N. A. (1987). A simple equation for description of solute release I. Fickian and non-Fickian release from non-swellable devices in form of slabs, sphere, cylinders or discs. Journal of Controlled Release, 5(1), 23-36. http:// dx.doi.org/10.1016/0168-3659(87)90034-4.

23. Karadag, E., Saraydin, D., \& Guven, O. (2001). Radiation induced superadsorbent hydrogels. Acrylamide/Itaconic acid copolymers. Macromolecular Materials and Engineering, 286(1), 34-42. http://dx.doi.org/10.1002/1439-2054(20010101)286:1<34::AIDMAME34>3.0.CO;2-J.

24. Bocourt, M., Bada, N., Acosta, N., Bucio, E., \& Peniche, C. (2014). Synthesis and characterization of novel $\mathrm{pH}$-sensitive chitosan-poly(acrylamide-co-itaconic acid) hydrogels. Polymer International, 63(9), 1715-1723. http://dx.doi.org/10.1002/ pi.4699.

25. Hu, D. S.-G., \& Lin, M. T. S. (1994). Water-polymer interactions and critical phenomena of swelling in inhomogeneous poly(acrylonitrile-acrylamide-acrylic acid) gels. Polymer, 35(20), 4416-4422. http://dx.doi.org/10.1016/0032-3861(94)90101-5.

26. Wang, J., Wu, W., \& Lin, Z. (2008). Kinetics and thermodynamics of the water sorption of 2-Hydroxyethyl methacrylate/styrene copolymer hydrogels. Journal of Applied Polymer Science, 109(5), 3018-3023. http://dx.doi.org/10.1002/app.28403.

27. Peniche, C., Cohen, M. E., Vázquez, B., \& San Román, J. (1997). Water sorption of flexible networks based on 2-hydroxyethyl methacrylate-triethylenglycol dimethacrylate copolymers. Polymer, 38(24), 5977-5982. http://dx.doi.org/10.1016/S00323861(96)01058-0.

28. Mark, J. E., \& Erman, B. (Ed.). (1988). Rubberlike elasticity: a molecular primer. New York: Wiley.

29. Flory, P. J., \& Rehner, J., Jr. (1943). Statistical mechanics of swelling of crosslinked polymer networks II. The Journal of Chemical Physics, 11, 521-526.

30. Teraoka, I. (2002). Polymer solutions: an introduction to physical properties. New York: Wiley.

31. Orwol, R. A., \& Chong, Y. S. (1999). Polyacrylamide. In J. E. Mark (Ed.), Polymer data handbook (pp. 247-251). Oxford: Oxford University Press.

32. Day, J. C., \& Robb, I. D. (1981). Thermodynamic parameters of polyacrylamides in water. Polymer, 22(11), 1530-1533. http://dx.doi.org/10.1016/0032-3861(81)90324-4.

33. Uzun, O., Hassnisaber, M., Şen, M., \& Guven, O. (2003). Enhancement and control of cross-linking of dimethylaminoethyl methacrylate irradiated at low dose rate in the presence of ethylene glycol dimethacrylate. Nuclear Instruments and Methods in Physics Research Section B, 208, 242-246. http:// dx.doi.org/10.1016/S0168-583X(03)01112-1.

Received: Feb. 03, 2014

Revised: May 25, 2015

Accepted: Aug. 15, 2015 